\title{
REVIEWS
}

\section{Ovarian Hyperstimulation Caused by Gonadotroph Pituitary Adenoma - Review}

${ }^{1}$ Department of Endocrinology, Diabetology and Isotope Therapy, Wroclaw Medical University, Poland

${ }_{2} 1^{\text {st }}$ Department of General, Gastroenterological and Endocrinological Surgery, Wroclaw Medical University, Poland

A - research concept and design; $\mathbf{B}$ - collection and/or assembly of data; $\mathbf{C}$ - data analysis and interpretation;

$\mathbf{D}$ - writing the article; $\mathbf{E}$ - critical revision of the article; $\mathbf{F}$ - final approval of article; $\mathbf{G}$ - other

\begin{abstract}
Ovarian hyperstimulation syndrome (OHSS) occurs mostly as an iatrogenic complication of assisted reproductive technology. Gonadotroph pituitary adenomas are rarely associated with OHSS. To the authors' knowledge, to date only 30 cases of spontaneous ovarian stimulation associated with gonadotroph adenomas have been reported in women and only 2 in children. The most common symptoms in such cases included menstrual disturbances, abdominal or pelvic pain, abdominal distension and increased girth. Galactorrhea, nausea and vomiting were also reported. Neurological symptoms occurred when the size of the pituitary tumor reached at least $20 \mathrm{~mm}$. Transvaginal ultrasound examination usually demonstrated enlarged multicystic ovaries. MRIs of the pituitary revealed macroadenomas up to $61 \mathrm{~mm}$ in maximum diameter. The hormonal profiles of the reported cases showed normal or elevated FSH levels, suppressed LH levels, elevated estradiol levels and supranormal concentrations of prolactin. Transsphenoidal surgery is the therapy of choice, however other treatment modalities can be utilized in selected cases (Adv Clin Exp Med 2015, 24, 4, 695-703).
\end{abstract}

Key words: ovarian hyperstimulation syndrome, pituitary adenoma, menstruation disturbances, gonadotropins.

Ovarian hyperstimulation syndrome (OHSS) occurs mostly as an iatrogenic complication of assisted reproductive technology [1]. Classification of OHSS is based on clinical features and laboratory findings. Symptoms vary from abdominal pain and bloating, nausea, vomiting and ovarian enlargement to possibly life-threatening conditions including renal failure, hypovolemic shock, adult respiratory distress syndrome, thromboembolism and pericardial effusion. As Mathur et al. pointed out, the cause of severe forms of OHSS is "an increase in vascular permeability resulting in a fluid shift from intravascular to third-space compartments" [2]. Vascular endothelial growth factor (VEGF) produced in stimulated ovaries under the influence of human chorionic gonadotropin (hCG) is thought to play a crucial role in the pathophysiology of OHSS [3]. Elevated VEGF blood levels correlate with the development of OHSS and its severity [4]. Spontaneous OHSS is rarely reported and usually associated with primary hypothyroidism, bilateral granulosa cell tumors and spontaneous pregnancy in women with polycystic ovary syndrome [5]. Follicle-stimulating hormone receptor gene polymorphisms might be responsible for modulating receptor sensitivity and susceptibility to OHSS [6].

To the authors' knowledge, only 32 cases of spontaneous ovarian stimulation induced by gonadotroph pituitary adenomas have been published (Table 1). Gonadotropinomas comprise $80-90 \%$ of the clinically nonfunctioning pituitary adenomas and account for $40-50 \%$ of all pituitary macroadenomas [7]. In vitro studies suggest that they are equally common in middle-aged men and women; however, they are difficult to diagnose in post-menopausal women because of physiological increases in the serum concentration of gonadotropins [8]. Gonadotroph adenomas rarely produce a recognizable clinical syndrome. They are 


\begin{tabular}{|c|c|c|c|c|c|c|c|c|c|c|}
\hline 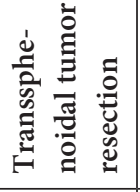 & + & + & + & + & + & + & + & + & + & + \\
\hline 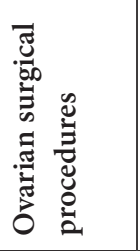 & 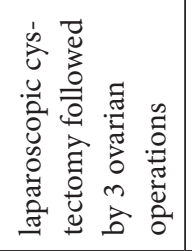 & 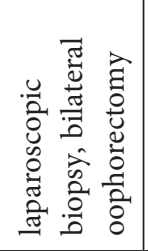 & 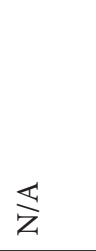 & 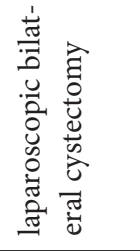 & 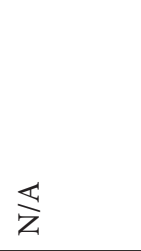 & 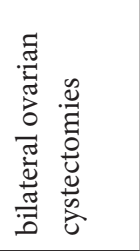 & 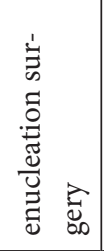 & $\overleftrightarrow{\longleftarrow}$ & 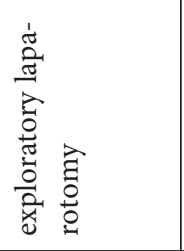 & 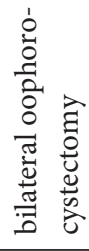 \\
\hline 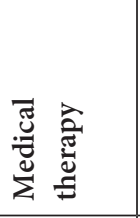 & 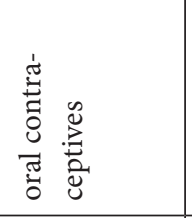 & 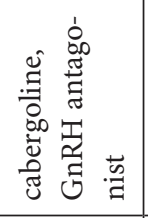 & $\overleftrightarrow{\mathbb{Z}}$ & 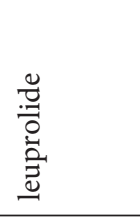 & 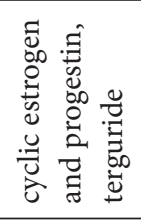 & $\underset{\mathbb{Z}}{\mathbb{Z}}$ & 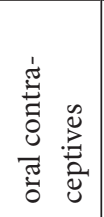 & 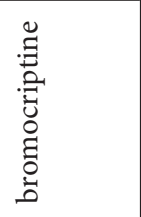 & 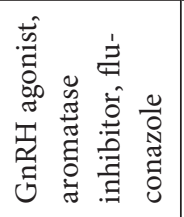 & $\underset{\mathbb{Z}}{\mathbb{Z}}$ \\
\hline 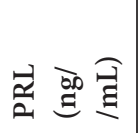 & $\begin{array}{l}\text { 竞 } \\
\text { in } \\
\hat{6}\end{array}$ & $\begin{array}{l}\text { I } \\
\text { Oे }\end{array}$ & $\begin{array}{l}\text { I } \\
\infty \\
+ \\
+ \\
-\end{array}$ & I & $\begin{array}{l}\frac{\pi}{2} \\
\stackrel{2}{2}\end{array}$ & $\frac{\pi}{\widehat{N}}$ & $\begin{array}{l}\Psi \\
0 \\
\infty \\
0 \\
\stackrel{0}{0} \\
i n\end{array}$ & $\begin{array}{l}\text { 垔 } \\
\text { Oे } \\
\text { in }\end{array}$ & 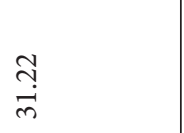 & $\stackrel{m}{m}$ \\
\hline 되 & $\begin{array}{l}\text { I } \\
\stackrel{9}{\circ} \\
\stackrel{0}{-}\end{array}$ & $\begin{array}{l} \pm \\
\hat{N} \\
\hat{0} \\
0\end{array}$ & 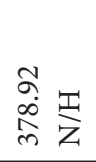 & $\begin{array}{l}\text { 吉 } \\
\text { 它 }\end{array}$ & 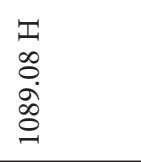 & 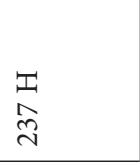 & $\begin{array}{l}z \\
\text { ț } \\
\text { n }\end{array}$ & $\begin{array}{l}\text { I } \\
8 \\
0 \\
\end{array}$ & $\begin{array}{l}\text { I } \\
\stackrel{1}{\Delta} \\
\infty\end{array}$ & 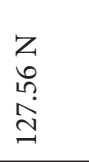 \\
\hline 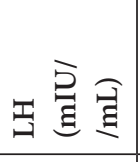 & $\begin{array}{l}\overrightarrow{0} \\
\stackrel{0}{0} \\
\end{array}$ & $\begin{array}{l}Z \\
\infty \\
i \\
\end{array}$ & $\begin{array}{l}+1 \\
\infty \\
0 \\
0\end{array}$ & in & 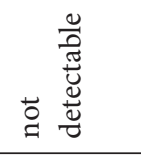 & $\overrightarrow{0}$ & $\begin{array}{l}\overrightarrow{1} \\
\stackrel{0}{0} \\
\mathrm{v}\end{array}$ & $\begin{array}{l}\underset{Z}{Z} \\
\underset{i}{ } \\
\end{array}$ & $\begin{array}{l}\overrightarrow{1} \\
\stackrel{0}{0} \\
0 \\
0\end{array}$ & $\begin{array}{l}\overrightarrow{1} \\
\stackrel{0}{0} \\
0 \\
\mathrm{v}\end{array}$ \\
\hline 昰总 & $\begin{array}{l}\stackrel{ \pm}{1} \\
\stackrel{2}{2}\end{array}$ & $\begin{array}{l}z \\
m \\
2\end{array}$ & $\begin{array}{l}Z \\
⿱ 亠 䒑 \\
\Xi \\
\end{array}$ & 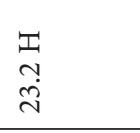 & $\begin{array}{l} \pm \\
m \\
0 \\
0\end{array}$ & $\begin{array}{l}z \\
\infty \\
0 \\
0 \\
\end{array}$ & $\begin{array}{l}z \\
\alpha \\
\alpha \\
o \\
\end{array}$ & $\begin{array}{l}z \\
\sigma \\
\sigma \\
\end{array}$ & \begin{tabular}{l}
$z$ \\
\multirow{2}{*}{} \\
$\stackrel{6}{6}$
\end{tabular} & 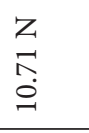 \\
\hline 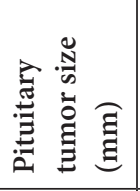 & $\begin{array}{l}\tilde{\lambda} \\
x \\
\hat{N} \\
x \\
\qquad \\
\qquad \\
\end{array}$ & $\begin{array}{l}\infty \\
\overrightarrow{1} \\
x \\
n \\
x \\
x \\
\\
\end{array}$ & 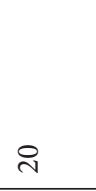 & 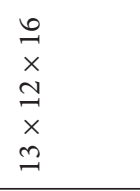 & 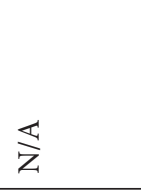 & $\stackrel{2}{\sim}$ & 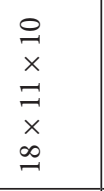 & $\begin{array}{l}q \\
\stackrel{+}{1} \\
\times \\
0 \\
\dot{m} \\
\times \\
\dot{m} \\
\end{array}$ & $\overleftrightarrow{\Delta}$ & $\overleftrightarrow{\mathbb{Z}}$ \\
\hline 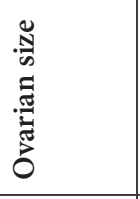 & $\sum_{i=1}$ & 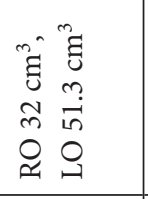 & $\sum_{\mid=1}$ & 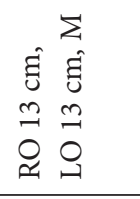 & $\Sigma$ & 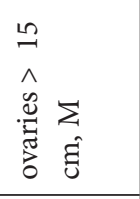 & $\sum_{4}$ & 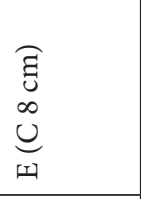 & $\sum_{i}$ & $\sum_{i=1}$ \\
\hline $\begin{array}{l}\text { ह } \\
\text { है } \\
\text { है: } \\
\text { हn }\end{array}$ & 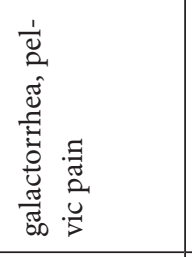 & 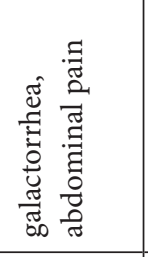 & 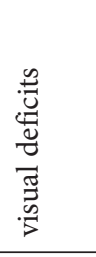 & 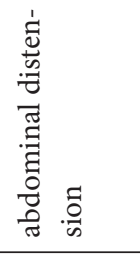 & 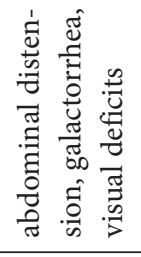 & 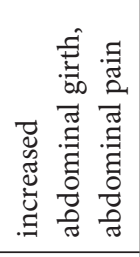 & 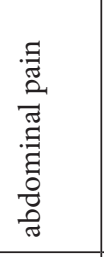 & 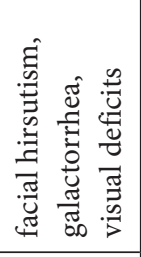 & $\overleftrightarrow{\mathrm{z}}$ & 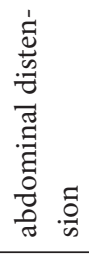 \\
\hline 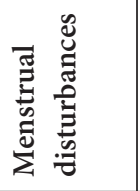 & 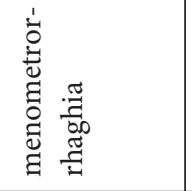 & 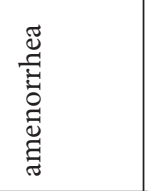 & $\begin{array}{l}\stackrel{\vec{G}}{\Xi} \\
\stackrel{\Xi}{\circ} \\
\Xi\end{array}$ & 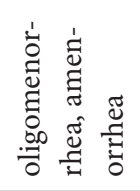 & 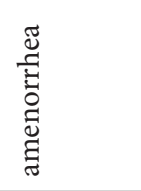 & 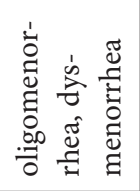 & 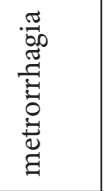 & 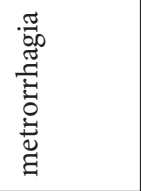 & 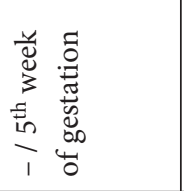 & 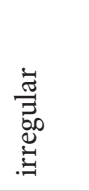 \\
\hline 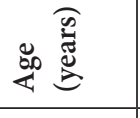 & 아 & $\stackrel{\sim}{*}$ & $\hat{\sim}$ & $\vec{m}$ & $\vec{\sim}$ & Әे & $\stackrel{n}{n}$ & m & $\tilde{n}$ & q \\
\hline 室 & 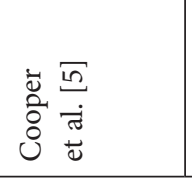 & 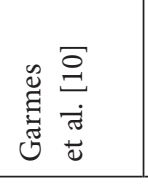 & 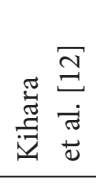 & 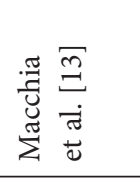 & 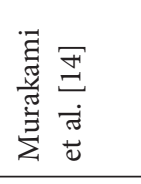 & 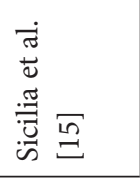 & 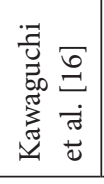 & 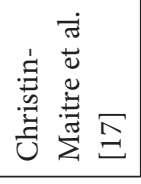 & 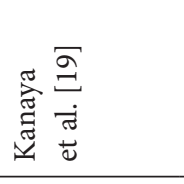 & \\
\hline
\end{tabular}




\begin{tabular}{|c|c|c|c|c|c|c|c|c|}
\hline 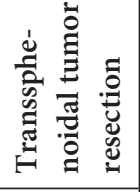 & + & + & + & + & + & + & $\overleftrightarrow{\mathrm{z}}$ & + \\
\hline 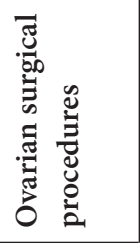 & 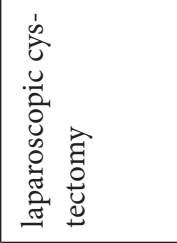 & $\overleftrightarrow{Z}$ & 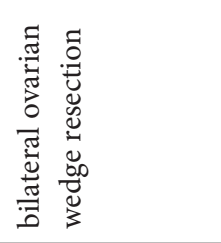 & 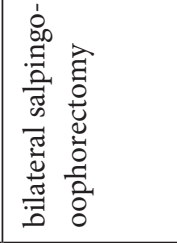 & 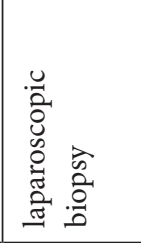 & 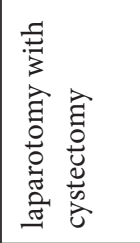 & $\frac{\mathbb{Z}}{\mathrm{Z}}$ & $\frac{\mathbb{Z}}{\mathrm{Z}}$ \\
\hline 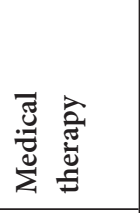 & 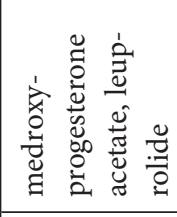 & 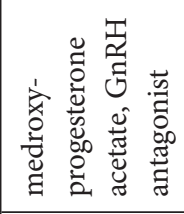 & 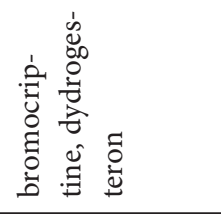 & 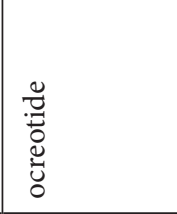 & 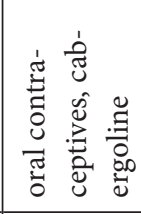 & 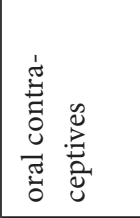 & 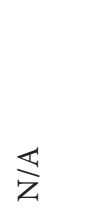 & 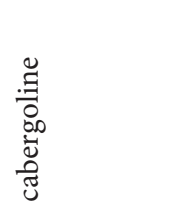 \\
\hline 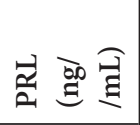 & $\overleftrightarrow{\mathbb{Z}}$ & $\begin{array}{l}\text { I } \\
\hat{0} \\
\text { in }\end{array}$ & $\stackrel{I}{R}$ & 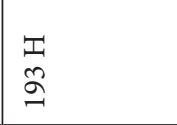 & $\underset{⿱}{i}$ & $\begin{array}{l}3 \\
6\end{array}$ & $\begin{array}{l}\underset{I}{N} \\
\stackrel{+}{i}\end{array}$ & $\begin{array}{l}7 \\
2 \\
2\end{array}$ \\
\hline 되 & 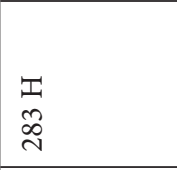 & $\begin{array}{l}1 \\
8 \\
8 \\
1 \\
1\end{array}$ & 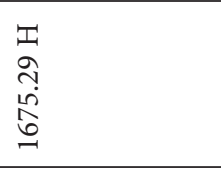 & 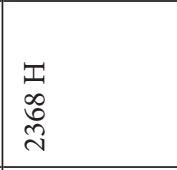 & $\begin{array}{l}z \\
z \\
\vdots \\
\vdots\end{array}$ & 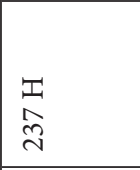 & $\begin{array}{l}\text { T } \\
\text { مे }\end{array}$ & \begin{tabular}{l}
1 \\
0 \\
\multirow{3}{*}{} \\
$\infty$ \\
$\infty$
\end{tabular} \\
\hline 出总訇 & $\vec{\jmath}$ & in & in & $\begin{array}{l}z \\
a \\
\dot{+}\end{array}$ & $\overrightarrow{\vec{\sigma}}$ & $\overrightarrow{0}$ & $\begin{array}{l}\overrightarrow{+} \\
\text { ọ } \\
\text { v }\end{array}$ & $\begin{array}{l}\vec{H} \\
\text { 옹 } \\
0 \\
\dot{0} \\
\mathrm{v}\end{array}$ \\
\hline 密总 & $\begin{array}{l}z \\
\text { n? } \\
n\end{array}$ & $\begin{array}{l}\stackrel{I}{1} \\
\stackrel{\infty}{0} \\
\stackrel{0}{=}\end{array}$ & $\begin{array}{l}\underset{I}{H} \\
\stackrel{\sim}{\rightarrow}\end{array}$ & $\begin{array}{l}\text { I } \\
\stackrel{1}{0} \\
\stackrel{1}{-}\end{array}$ & 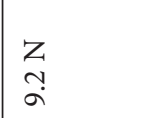 & $\begin{array}{l}z \\
0 \\
\infty \\
b\end{array}$ & $\begin{array}{l}z \\
z \\
\infty \\
\infty\end{array}$ & 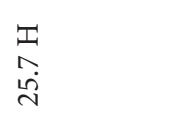 \\
\hline 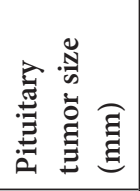 & 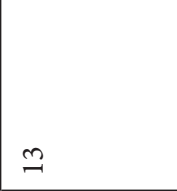 & $\exists$ & $\begin{array}{l}\stackrel{N}{1} \\
\times \\
\infty \\
\text { in }\end{array}$ & 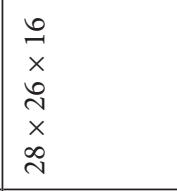 & in & 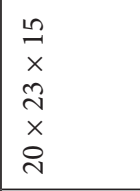 & $a$ & 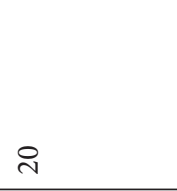 \\
\hline 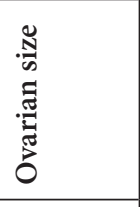 & 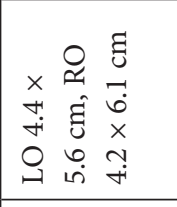 & $\Sigma$ & 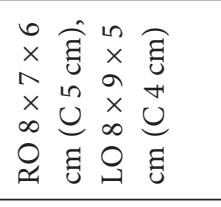 & 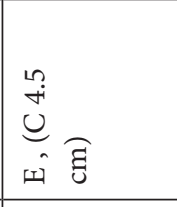 & 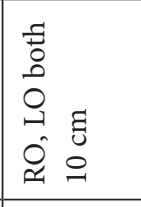 & 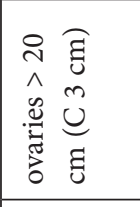 & $\sum_{i=1}$ & 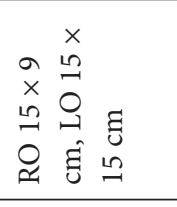 \\
\hline 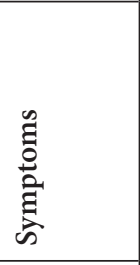 & 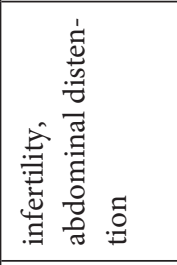 & 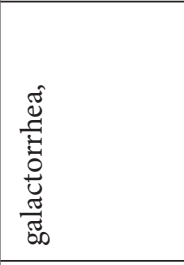 & 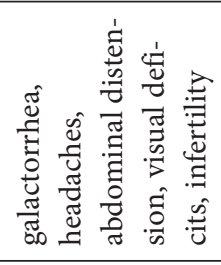 & 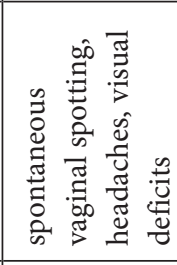 & 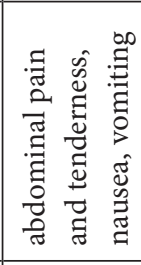 & 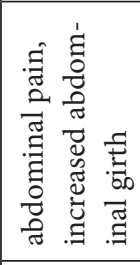 & 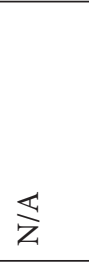 & 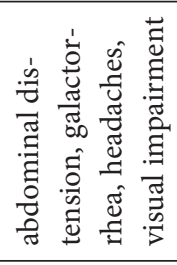 \\
\hline 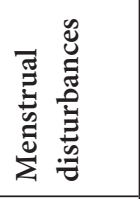 & 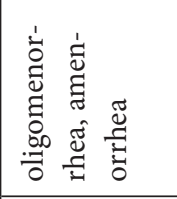 & 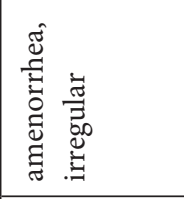 & 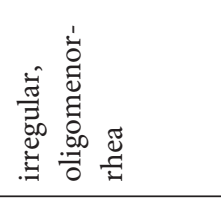 & 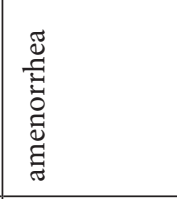 & 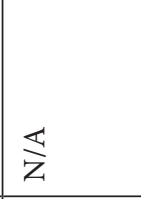 & 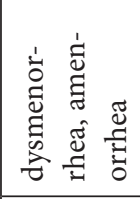 & 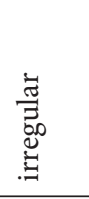 & 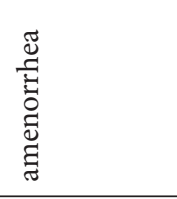 \\
\hline 总总 & $\stackrel{n}{m}$ & సે & $\ddot{\sim}$ & $\hat{m}$ & $\stackrel{\infty}{\sim}$ & 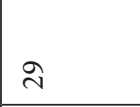 & 아 & $\stackrel{m}{\rightarrow}$ \\
\hline 害 & 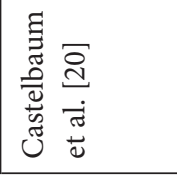 & 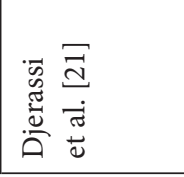 & 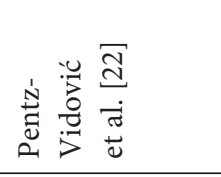 & 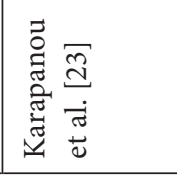 & 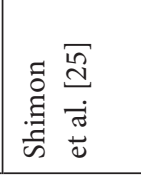 & 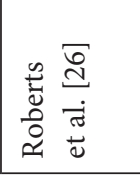 & 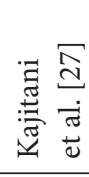 & 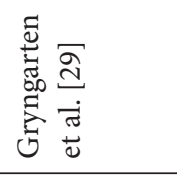 \\
\hline
\end{tabular}




\begin{tabular}{|c|c|c|c|c|c|c|c|}
\hline 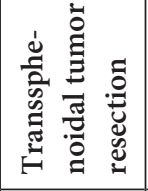 & $\overleftrightarrow{⿱ 乛}$ & + & + & + & + & + & $\overleftrightarrow{\breve{z}}$ \\
\hline 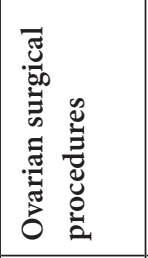 & $\overleftrightarrow{⿱ 乛}$ & $\overleftrightarrow{\breve{z}}$ & $\overleftrightarrow{z}$ & 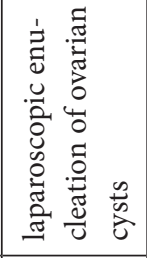 & 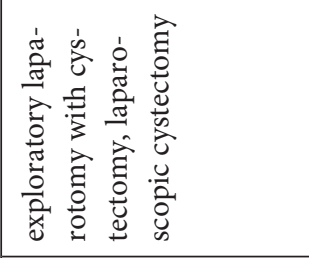 & 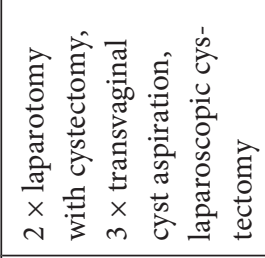 & 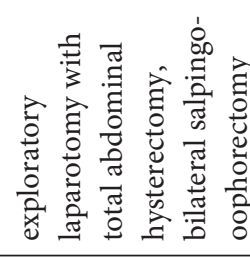 \\
\hline 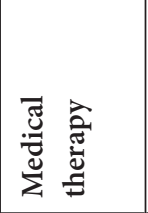 & 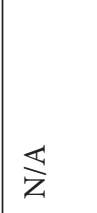 & 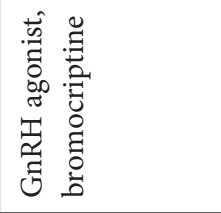 & $\overleftrightarrow{⿱ 亠 乂}$ & 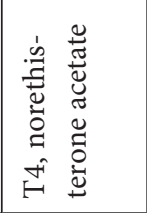 & 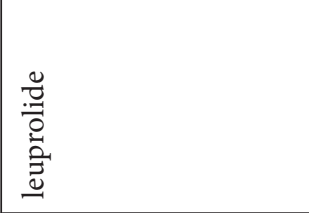 & 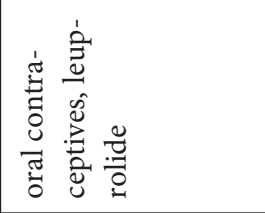 & $\frac{\pi}{z}$ \\
\hline 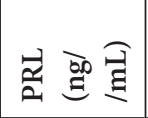 & $\begin{array}{l}\text { I } \\
\stackrel{1}{+} \\
\stackrel{+}{\sim}\end{array}$ & $\begin{array}{l}\stackrel{I}{+} \\
\stackrel{+}{\dot{m}}\end{array}$ & $\begin{array}{l}\text { I } \\
\text { 范 }\end{array}$ & 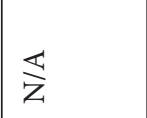 & 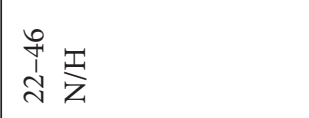 & $\begin{array}{l}\text { I } \\
\infty \\
i n\end{array}$ & 工 \\
\hline 외 & $\begin{array}{l}\text { 崩 } \\
\text { مे }\end{array}$ & 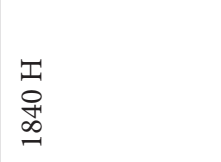 & 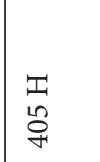 & 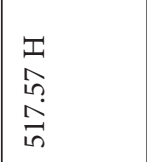 & 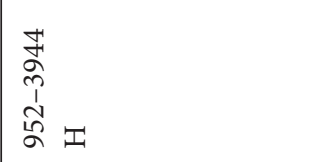 & 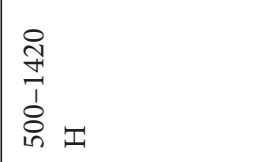 & $\begin{array}{l}1 \\
0 \\
6\end{array}$ \\
\hline 出总宝 & $\begin{array}{l}\sqrt{1} \\
\text { ָे } \\
\text { v }\end{array}$ & in & $\vec{a}$ & ले & $\vec{v}$ & $\vec{v}$ & ـــ \\
\hline 密总䒠 & $\begin{array}{l}Z \\
\infty \\
\infty \\
\infty \\
\end{array}$ & 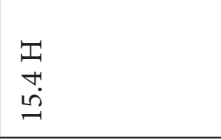 & 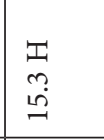 & $\begin{array}{l}z \\
\text { a } \\
+\end{array}$ & $\begin{array}{l}I \\
\pm \\
I \\
=\end{array}$ & $\begin{array}{l}I \\
\infty \\
I \\
\pm \\
\pm\end{array}$ & $\overleftrightarrow{\Xi}$ \\
\hline 总 & $a$ & $\wedge$ & $\overleftrightarrow{\Xi}$ & $\Xi$ & $a$ & 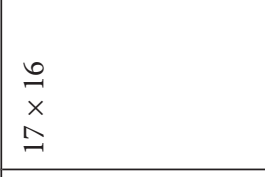 & $\vec{b}$ \\
\hline 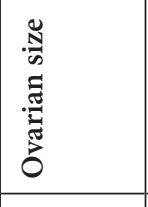 & $\sum_{\mid=1}$ & 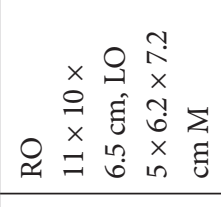 & 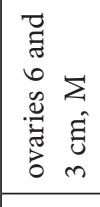 & 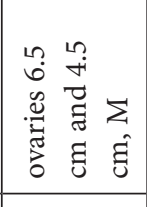 & 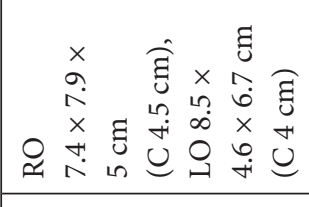 & 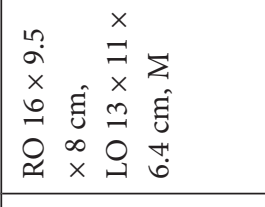 & 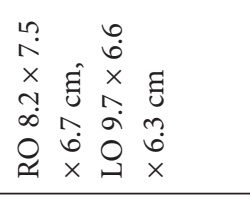 \\
\hline 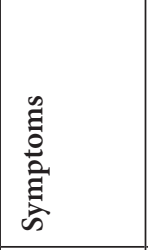 & 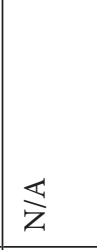 & 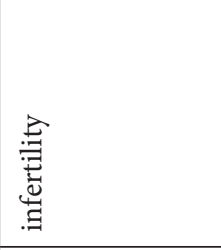 & $\overleftrightarrow{\Xi}$ & 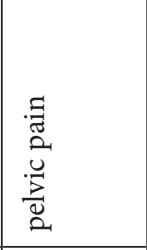 & 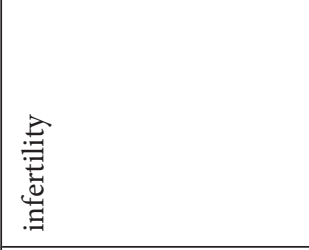 & 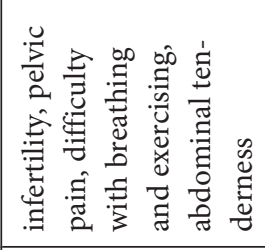 & 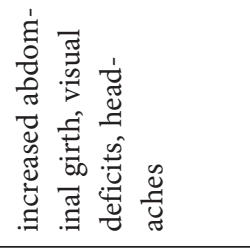 \\
\hline 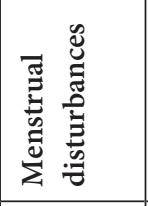 & 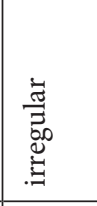 & 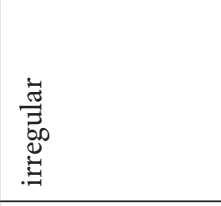 & 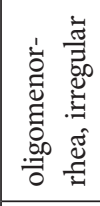 & 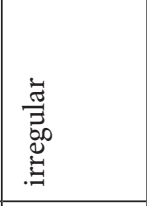 & 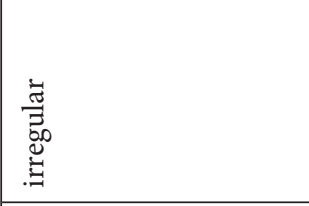 & 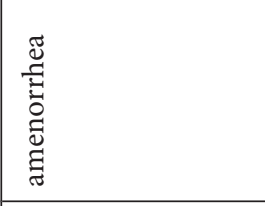 & 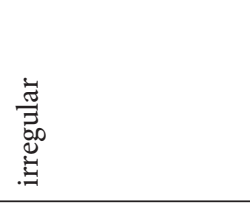 \\
\hline 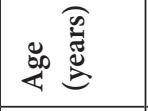 & 우 & సे & F & $\stackrel{\infty}{\sim}$ & $\vec{m}$ & ి & $\stackrel{\mathscr{F}}{*}$ \\
\hline 恋 & 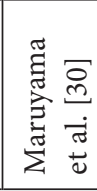 & 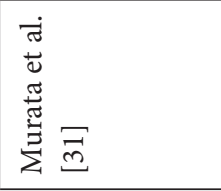 & 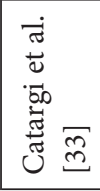 & 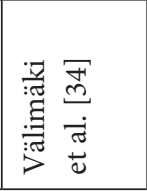 & 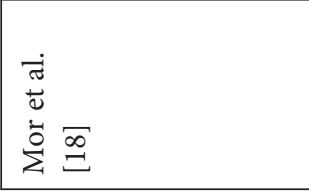 & & \\
\hline
\end{tabular}




\begin{tabular}{|c|c|c|c|c|c|c|c|}
\hline 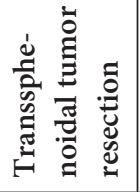 & + & + & + & + & + & + & + \\
\hline 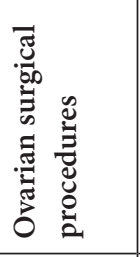 & $\overleftrightarrow{\mathrm{Z}}$ & 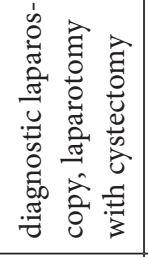 & $\overleftrightarrow{\mathrm{z}}$ & 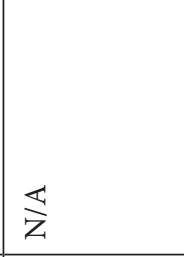 & $\overleftrightarrow{\Xi}$ & $\frac{\overleftrightarrow{z}}{\bar{z}}$ & $\frac{\overleftrightarrow{z}}{\bar{z}}$ \\
\hline 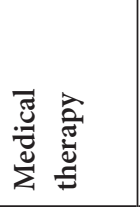 & 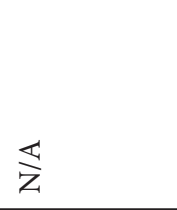 & $\frac{\mathbb{Z}}{\mathrm{z}}$ & $\underset{\mathrm{z}}{\mathbb{Z}}$ & $\frac{\mathbb{Z}}{\mathrm{z}}$ & 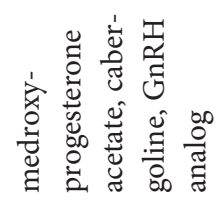 & 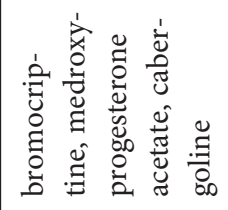 & 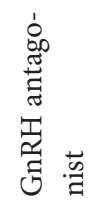 \\
\hline 总总 $\widehat{\Xi}$ & 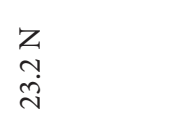 & $\begin{array}{l}z \\
-\dot{m} \\
=\end{array}$ & $\underset{\mathrm{Z}}{\overleftrightarrow{\mathrm{z}}}$ & $\begin{array}{l}\text { 焉 } \\
\text { in }\end{array}$ & $\begin{array}{l}\mathbb{\Psi} \\
\stackrel{\infty}{\wedge}\end{array}$ & 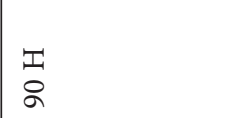 & $\begin{array}{l}z \\
\stackrel{2}{n} \\
\end{array}$ \\
\hline 쇠 & 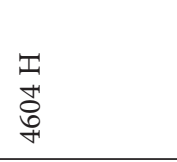 & 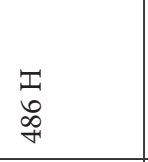 & $\begin{array}{l}\text { I } \\
\text { 岁 } \\
i n\end{array}$ & $\begin{array}{l}1 \\
0 \\
0 \\
\text { o } \\
\text { m }\end{array}$ & 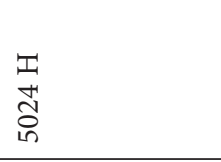 & 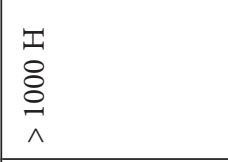 & $\begin{array}{l}\Psi \\
n \\
n \\
n \\
n\end{array}$ \\
\hline 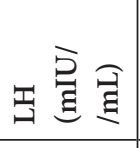 & $\vec{a}$ & $\begin{array}{l}\rightarrow \\
\stackrel{\infty}{0} \\
0\end{array}$ & $\begin{array}{l}\vec{A} \\
\hat{0}\end{array}$ & $\begin{array}{l}u \\
10 \\
0 \\
v\end{array}$ & $\begin{array}{l}Z \\
m \\
-\end{array}$ & $\begin{array}{l}z \\
m \\
i\end{array}$ & 송 \\
\hline 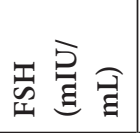 & $\begin{array}{l}Z \\
\substack{Z \\
\infty \\
\infty}\end{array}$ & $\overrightarrow{\text { i }}$ & $\begin{array}{l}\underset{I}{1} \\
\stackrel{\infty}{=} \\
=\end{array}$ & $\begin{array}{l}\text { I } \\
\stackrel{\infty}{m} \\
\text { m. }\end{array}$ & $\begin{array}{l}z \\
\underset{+}{Z}\end{array}$ & $\begin{array}{l}1 \\
\infty \\
\infty \\
\\
-1 \\
\end{array}$ & $\begin{array}{l}z \\
b \\
\infty \\
\infty\end{array}$ \\
\hline 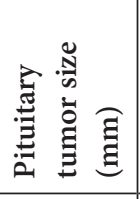 & $\begin{array}{l}\stackrel{\infty}{1} \\
\times \\
\stackrel{1}{x} \\
\times \\
\infty \\
\stackrel{-}{\longrightarrow}\end{array}$ & 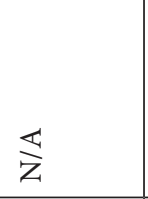 & $\stackrel{\infty}{\sim}$ & $\overleftrightarrow{\Xi}$ & 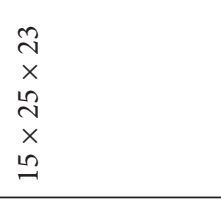 & $\begin{array}{l}\tilde{N} \\
\times \\
\stackrel{+}{+}\end{array}$ & $\infty$ \\
\hline 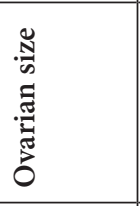 & 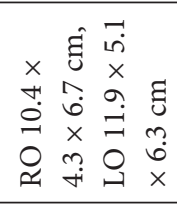 & 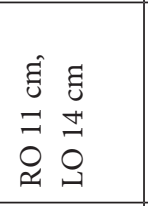 & $\Sigma$ & 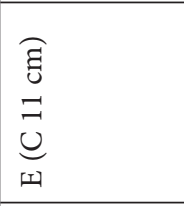 & 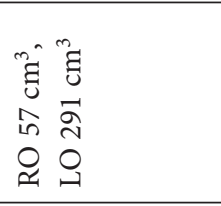 & 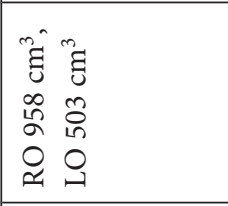 & 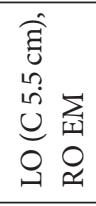 \\
\hline 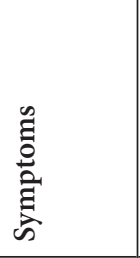 & 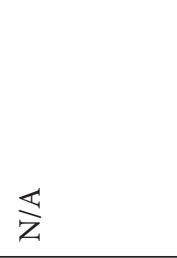 & 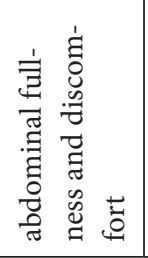 & 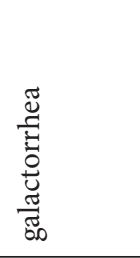 & 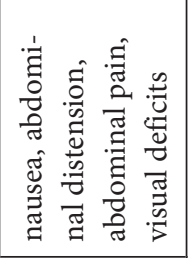 & 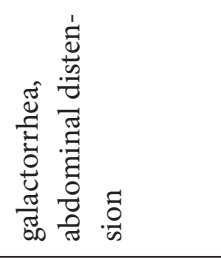 & 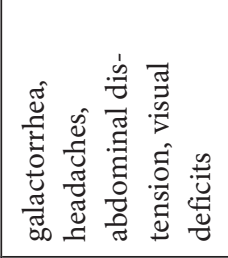 & 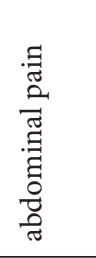 \\
\hline 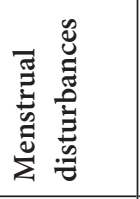 & 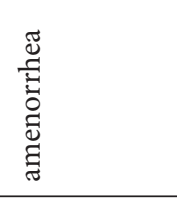 & 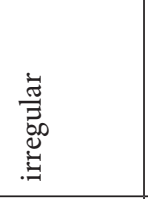 & 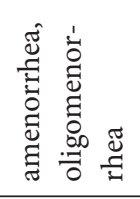 & 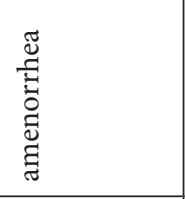 & 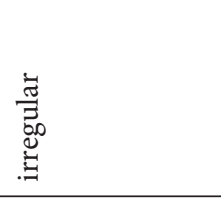 & 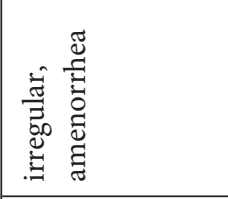 & 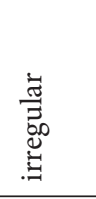 \\
\hline 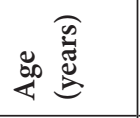 & ঐे & i & ले & 으 & $\stackrel{\infty}{m}$ & 우 & $\stackrel{\infty}{\sim}$ \\
\hline È & 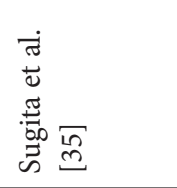 & 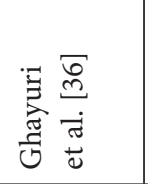 & 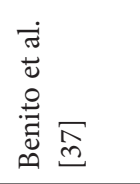 & 兽离 & \multicolumn{2}{|l|}{ 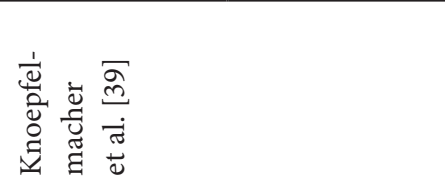 } & 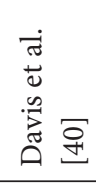 \\
\hline
\end{tabular}


usually revealed by symptoms due to mass effects such as visual impairment, headaches and hormonal deficiencies [9].

\section{Clinical Presentation}

To date, 30 cases of spontaneous ovarian stimulation associated with gonadotroph adenomas have been reported in women and only two in children. Clinical manifestations vary from precocious puberty to OHSS. The patients' age ranged from 10 to 43 years. Menstrual disorders were the main reason medical attention was sought. The most common disturbances included amenorrhea, oligomenorrhea and irregular menses. Metrorrhagia and menometrorrhagia have been mentioned in only three papers. Other complaints were abdominal or pelvic pain, abdominal distension and increased girth, and difficulties in conceiving. Less common findings were galactorrhea, nausea and vomiting. Neurological symptoms occurred when the size of the pituitary tumor reached $20 \mathrm{~mm}$ or more in diameter. Deficits in visual field examinations were disclosed in 7 patients. Bitemporal hemianopsia was the most common defect. Additionally, two patients experienced loss of visual acuity. Growth hormone deficiency was noted at an initial examination in one case. Pituitary imaging almost always depicted a macroadenoma. The size of the tumors in the reviewed cases ranged from $7 \mathrm{~mm}$ to $61 \mathrm{~mm}$ in maximum diameter (Table 1).

Immunohistochemical techniques are required to confirm a diagnosis of gonadotroph pituitary adenoma [10]. Immunostaining of the resected tumor tissue can be positive even when there is no measurable hormone secretion $[11,12]$. Ultrasound examination usually revealed multicystic enlarged ovaries, similar to the ovaries seen in OHSS resulting from exogenous gonadotropin administration $[13,14]$. Sicilia et al. reported a case of a 29-year-old woman who presented with acute abdomen caused by ovarian torsion. The ovaries were multicystic and measured $>15 \mathrm{~cm}$ [15]. In the reviewed cases, the largest depicted ovary measured $20 \mathrm{~cm}$ in diameter (Table 1). In most of the reported cases ascites was not detected. The literature suggests that ascites is attributable to iatrogenic OHSS, which makes it a distinguishing feature of this entity $[13,16,17]$. Nonetheless, a small volume of ascites has been found in a few cases of women with developed ovarian hyperstimulation $[14,18]$. Kanaya et al. described the case of a 32-year-old woman who became pregnant naturally and developed severe OHSS in the $5^{\text {th }}$ week of gestation. After the termination of the pregnancy the ascites resolved but multicystic ovarian enlargement emerged again. The authors stated that FSH stimulation itself is not sufficient to provoke ascites, and that LH or hCG stimulation is required in addition [19]. Menstrual disturbances, infertility and multiple ovarian cysts may resemble polycystic ovary syndrome (PCOS). However, ovarian follicles in women with PCOS are rarely larger than $10 \mathrm{~mm}$.

Another important feature in differential diagnosis is the fact that the FSH/LH ratio is usually decreased in PCOS and elevated in subjects with pituitary tumors [20]. The hormonal profiles in the reported cases of OHSS induced by gonadotroph pituitary adenomas showed normal or increased FSH levels (normal FSH in 17 out of 31 cases; elevated FSH in 14 out of 31 cases), suppressed LH levels (27 out of 32 cases), increased estradiol levels ( 29 out of 32 cases) and supranormal concentrations of prolactin (24 out of 27 cases). In a few cases $\alpha$-subunit concentrations were estimated. Elevated $\alpha$-subunit levels may indicate the presence of pituitary adenoma $[8,9]$. FSH secreted by the tumor cells causes the formation of the multiple ovarian cysts and may considerably increase the production of estradiol [21, 22]. In some reports high estradiol levels contributed to the development of endometrial hyperplasia $[17,21,23]$. As a result of the negative feedback mechanism, FSH may remain within the normal range. However, in almost half of the reviewed cases FSH levels were elevated, which supports the theory of impaired negative feedback mechanism [24]. Normal FSH concentrations are capable of inducing OHSS, probably because of enhanced FSH bioactivity $[25,26]$. Kajitani et al. found that the serum FSH bioactivity/immunoactivity ratio in a patient with gonadotroph adenoma was "mostly equal" to the ratios found in two healthy women. Therefore, a mechanism other than enhanced bioactivity may be responsible for the development of OHSS [27]. These findings were consistent with Kanaya's study [19]. The two-cell, two-gonadotropin theory states that FSH and LH are both required to stimulate the ovaries to secrete estrogens. LH stimulates the theca cells to produce androgens, which are then transported to the granulosa cells and aromatized to estrogens under the influence of FSH $[17,25]$. However, in most of the reported cases LH levels were decreased. It is presumed that such low levels are sufficient to produce estradiol [19]. LH levels below the reference range are explained by estrogen-induced negative feedback mechanism or by the compression of normal pituitary tissue $[21,25]$. Other suggested mechanisms responsible for suppressed LH levels involve inhibition of pituitary LHRH receptors or 
inhibition of LHRH secretion from the hypothalamus due to an abnormal form of FSH [22]. In postmenopausal women gonadotropinomas are not recognizable unless suppressed LH levels are detected [28]. In women over the age of 45 years ovaries are not sensitive to FSH stimulation. Thus, the clinical picture does not involve OHSS [21]. In patients with gonadotroph adenomas a paradoxical responses of $\mathrm{LH}, \mathrm{FSH}$ or their subunits to thyrotropin releasing hormone (TRH) stimulation tests is found even if basal levels of these hormones are normal $[8,9,29,30]$. This test can be utilized in the diagnosis [31]. It has to be emphasized that the TRH test is not always positive $[25,32]$. The gonadotropin-releasing hormone $(\mathrm{GnRH})$ test is also used in the diagnosis $[12,13,33]$.

Hyperprolactinemia, which is noted in most cases of gonadotroph pituitary adenoma, may be caused by pituitary stalk compression or high estradiol concentrations [12, 21, 26]. Elevated prolactin levels are sometimes the first discovered hormonal disorder. An MRI performed because of increased prolactin levels can reveal the presence of a pituitary adenoma $[13,25]$.

\section{Treatment}

Transsphenoidal tumor resection is the approach of choice. Regression of enlarged multicystic ovaries, restoration of regular menstrual cycles and normalization in hormone levels can be observed after this surgery $[5,13]$. The paradoxical responses of FSH, LH and their subunits to TRH stimulation test are no longer present postoperatively $[12,34]$. Spontaneous pregnancies have also been observed in a few reports [18, 35, 36]. Castelbaum et al. reported a case of a woman who became pregnant ten months after the excision of a gonadotroph adenoma [20]. Patients who have undergone operations should be closely monitored due to the risk of pituitary insufficiency and diabetes insipidus [17, 21,37]. Recurrent and remnant tumors were common, occurring in 11 out of 29 cases; 4 patients therefore underwent reoperation. Radiotherapy and radiosurgery are used in the management of the residual or recurrent tumors $[7,17,21,23,26,37,38]$.

In general, medical therapy is thought to be ineffective $[5,13]$. Gonadotropin-releasing hormone agonists are not recommended. Administration of leuprolide resulted in elevation of estradiol and FSH levels and further ovarian cyst enlargement after one month of treatment [20]. Exacerbation of ovarian hyperstimulation during GnRH agonist therapy has also been noticed by other authors [19], which may suggest the presence of a pituitary gonadotroph adenoma [20]. In one case, $\mathrm{GnRH}$ analog administration resulted in a slight increase in tumor size [39]. Macchia et al. reported that long-acting leuprolide injection neither reduced serum estradiol and FSH levels nor prevented a rapid relapse of OHSS [13]. Furthermore, the first dose of leuprolide can induce pituitary apoplexy in a patient with asymptomatic gonadotroph adenoma [40].

Data on treatment with gonadotropin-releasing hormone antagonists is limited. In one report, a 30-day treatment using a GnRH antagonist and cabergoline resulted in a decrease in serum estradiol levels and improvement in the patient's clinical condition, with considerable reduction in ovarian volume. After GnRH antagonist cessation, estradiol levels increased and ultrasound disclosed further ovarian enlargement [10]. However, in another study, 4-week $\mathrm{GnRH}$ antagonist therapy did not normalize FSH, LH and $\alpha$-subunit concentrations [21].

Dopamine agonists, such as bromocriptine and cabergoline, can effectively reduce prolactin levels $[10,17,25,29,39]$. Additionally, cabergoline therapy can decrease estradiol and FSH levels [29]. Ovarian size reduction and suppression of estradiol levels were observed after the addition of cabergoline to medroxyprogesterone acetate treatment [39]. In the case of a 40-year old woman, bromocriptine administration restored regular menstrual cycles and resolved galactorrhea [39]. Somatostatin analogs might be also taken into consideration in selected cases [23].

\section{Conclusions}

Gonadotroph pituitary can be a cause of ovarian hyperstimulation. The clinical presentation commonly includes menstrual disturbances and multicystic ovarian enlargement. In such cases evaluation of concentrations of estradiol and gonadotropins is recommended. The typical endocrine profile reveals supranormal estradiol levels, normal or elevated FSH and suppressed LH values, which should be an indication for pituitary imaging. Transsphenoidal surgery is the treatment of choice. A detailed examination facilitates the proper diagnosis, thus unnecessary ovarian surgery can be avoided. 


\section{References}

[1] Kumar P, Sait SF, Sharma A, Kumar M: Ovarian hyperstimulation syndrome. J Hum Reprod Sci 2011, 4, 70-75.

[2] Mathur R, Kailasam C, Jenkins J: Review of the evidence base of strategies to prevent ovarian hyperstimulation syndrome. Hum Fertil (Camb) 2007, 10, 75-85.

[3] Elchalal U, Schenker JG: The pathophysiology of ovarian hyperstimulation syndrome - views and ideas. Hum Reprod 1997, 12, 1129-1137.

[4] Abramov Y, Barak V, Nisman B, Schenker JG: Vascular endothelial growth factor plasma levels correlate to the clinical picture in severe ovarian hyperstimulation syndrome. Fertil Steril 1997, 67, 261-265.

[5] Cooper O, Geller JL, Melmed S: Ovarian hyperstimulation syndrome caused by an FSH-secreting pituitary adenoma. Nat Clin Pract Endocrinol Metab 2008, 4, 234-238.

[6] Smits G, Olatunbosun O, Delbaere A, Pierson R, Vassart G, Costagliola S: Ovarian hyperstimulation syndrome due to a mutation in the follicle-stimulating hormone receptor. N Engl J Med 2003, 349, 760-766.

[7] Chaidarun SS, Klibanski A: Gonadotropinomas. Semin Reprod Med 2002, 20, 339-348.

[8] Daneshdoost L, Gennarelli TA, Bashey HM, Savino PJ, Sergott RC, Bosley TM, Snyder PJ: Recognition of gonadotroph adenomas in women. N Engl J Med 1991, 324, 589-594.

[9] Snyder PJ: Gonadotroph cell adenomas of the pituitary. Endocr Rev 1985, 6, 552-563.

[10] Garmes HM, Grassiotto OR, Fernandes YB, Queiroz L de S, Vassalo J, de Oliveira DM, Benneti-Pinto CL: A pituitary adenoma secreting follicle-stimulating hormone with ovarian hyperstimulation: treatment using a gonadotropin-releasing hormone antagonist. Fertil Steril 2012, 97, 231-234.

[11] Arafah BM, Nasrallah MP: Pituitary tumors: pathophysiology, clinical manifestations and management. Endocr Relat Cancer 2001, 8, 287-305.

[12] Kihara M, Sugita T, Nagai Y, Saeki N, Tatsuno I, Seki K: Ovarian hyperstimulation caused by gonadotroph cell adenoma: a case report and review of the literature. Gynecol Endocrinol 2006, 22, 110-113.

[13] Macchia E, Simoncini T, Raffaelli V, Lombardi M, Iannelli A, Martino E: A functioning FSH-secreting pituitary macroadenoma causing an ovarian hyperstimulation syndrome with multiple cysts resected and relapsed after leuprolide in a reproductive-aged woman. Gynecol Endocrinol 2012, 28, 56-59.

[14] Murakami T, Higashitsuji H, Yoshinaga K, Terada Y, Ito K, Ikeda H: Management of ovarian hyperstimulation due to follicle-stimulating hormone-secreting gonadotroph adenoma. BJOG 2004, 111, 1297-1300.

[15] Sicilia V, Earle J, Mezitis SGE: Multiple ovarian cysts and oligomenorrhea as the initial manifestations of a gonadotropin-secreting pituitary macroadenoma. Endocr Pract 2006, 12, 417-421.

[16] Kawaguchi T, Ogawa Y, Ito K, Watanabe M, Tominaga T: Follicle-stimulating hormone-secreting pituitary adenoma manifesting as recurrent ovarian cysts in a young woman - latent risk of unidentified ovarian hyperstimulation: a case report. BMC Res Notes 2013, 6, 408.

[17] Christin-Maitre S, Rongières-Bertrand C, Kottler ML, Lahlou N, Frydman R, Touraine P, Bouchard P: A spontaneous and severe hyperstimulation of the ovaries revealing a gonadotroph adenoma. J Clin Endocrinol Metab 1998, 83, 3450-3453.

[18] Mor E, Rodi IA, Bayrak A, Paulson RJ, Sokol RZ: Diagnosis of pituitary gonadotroph adenomas in reproductiveaged women. Fertil Steril 2005, 84, 757.

[19] Kanaya M, Baba T, Kitajima Y, Ikeda K, Shimizu A, Morishita M, Honnma H, Endo T, Saito T: Continuous follicle-stimulating hormone exposure from pituitary adenoma causes periodic follicle recruitment and atresia, which mimics ovarian hyperstimulation syndrome. Int J Womens Health 2012, 4, 427-431.

[20] Castelbaum AJ, Bigdeli H, Post KD, Freedman MF, Snyder PJ: Exacerbation of ovarian hyperstimulation by leuprolide reveals a gonadotroph adenoma. Fertil Steril 2002, 78, 1311-1313.

[21] Djerassi A, Coutifaris C, West VA, Asa SL, Kapoor SC, Pavlou SN, Snyder PJ: Gonadotroph adenoma in a premenopausal woman secreting follicle-stimulating hormone and causing ovarian hyperstimulation. J Clin Endocrinol Metab 1995, 80, 591-594.

[22] Pentz-Vidovíc I, SkorićT, Grubisić G, Korsíc M, Ivicevic-Bakulic T, Besenski N, Paladino J, Plavsíć V, Zarkovíć K: Evolution of clinical symptoms in a young woman with a recurrent gonadotroph adenoma causing ovarian hyperstimulation. Eur J Endocrinol 2000, 143, 607-614.

[23] Karapanou O, Tzanela M, Tamouridis N, Tsagarakis S: Gonadotroph pituitary macroadenoma inducing ovarian hyperstimulation syndrome: successful response to octreotide therapy. Hormones (Athens) 2012, 11, $199-202$.

[24] Lania A, Gangi E, Romoli R, Losa M, Travaglini P, Meringolo D, Ambrosi B, Faglia G, Beck-Peccoz P, Spada A: Impaired estrogen-induced negative feedback on gonadotropin secretion in patients with gonadotropin-secreting and nonfunctioning pituitary adenomas. Eur J Clin Invest 2002, 32, 335-340.

[25] Shimon I, Rubinek T, Bar-Hava I, Nass D, Hadani M, Amsterdam A, Harel G: Ovarian hyperstimulation without elevated serum estradiol associated with pure follicle-stimulating hormone-secreting pituitary adenoma. J Clin Endocrinol Metab 2001, 86, 3635-3640.

[26] Roberts JE, Spandorfer S, Fasouliotis SJ, Lin K, Rosenwaks Z: Spontaneous ovarian hyperstimulation caused by a follicle-stimulating hormone-secreting pituitary adenoma. Fertil Steril 2005, 83, 208-210.

[27] Kajitani T, Liu S, Maruyama T, Uchida H, Sakurai R, Masuda H, Nagashima T, Ono M, Arase T, Yoshimura Y: Analysis of serum FSH bioactivity in a patient with an FSH-secreting pituitary microadenoma and multicystic ovaries: A case report. Hum Reprod 2008, 23, 435-439.

[28] Wide L, Lundberg PO: Hypersecretion of an abnormal form of follicle-stimulating hormone associated with suppressed luteinizing hormone secretion in a woman with a pituitary adenoma. J Clin Endocrinol Metab 1981, 53, 923-930. 
[29] Gryngarten MG, Braslavsky D, Ballerini MG, Ledesma J, Ropelato MG, Escobar ME: Spontaneous ovarian hyperstimulation syndrome caused by a follicle-stimulating hormone-secreting pituitary macroadenoma in an early pubertal girl. Horm Res Paediatr 2010, 73, 293-298.

[30] Maruyama T, Masuda H, Uchida H, Nagashima T, Yoshimura Y: Follicle stimulating hormone-secreting pituitary microadenoma with fluctuating levels of ovarian hyperstimulation. Obstet Gynecol 2005, 105, 1215-1218.

[31] Murata Y, Ando H, Nagasaka T, Takahashi I, Saito K, Fukugaki H, Matsuzawa K, Mizutani S: Successful pregnancy after bromocriptine therapy in an anovulatory woman complicated with ovarian hyperstimulation caused by follicle-stimulating hormone-producing plurihormonal pituitary microadenoma. J Clin Endocrinol Metab 2003, 88, 1988-1993.

[32] Chanson P: Adénomes hypophysaires gonadotropes. Ann Endocrinol 2000, 61, 258-268.

[33] Catargi B, Felicie-Dellan E, Tabarin A: Comment on gonadotroph adenoma causing ovarian hyperstimulation. J Clin Endocrinol Metab 1999, 84, 3404.

[34] Välimäki MJ, Tiitinen A, Alfthan H, Paetau A, Poranen A, Sane T, Stenman U: Ovarian hyperstimulation caused by gonadotroph adenoma secreting follicle-stimulating hormone in 28 -year-old woman. J Clin Endocrinol Metab 1999, 84, 4204-4208.

[35] Sugita T, Seki K, Nagai Y, Saeki N, Yamaura A, Ohigashi S, Iwata A, Tatsuno I, Sekiya S: Successful pregnancy and delivery after removal of gonadotrope adenoma secreting follicle-stimulating hormone in a 29-year-old amenorrheic woman. Gynecol Obstet Invest 2005, 59, 138-143.

[36] Ghayuri M, Liu JH: Ovarian hyperstimulation syndrome caused by pituitary gonadotroph adenoma secreting follicle-stimulating hormone. Obstet Gynecol 2007, 109, 547-549.

[37] Benito M, Asa SL, Livolsi VA, West VA, Snyder PJ: Gonadotroph tumor associated with multiple endocrine neoplasia type 1. J Clin Endocrinol Metab 2005, 90, 570-574.

[38] Tashiro H, Katabuchi H, Ohtake H, Kaku T, Ushio Y, Okamura H: A follicle-stimulating hormone-secreting gonadotroph adenoma with ovarian enlargement in a 10-year-old girl. Fertil Steril 1999, 72, 158-160.

[39] Knoepfelmacher M, Danilovic DLS, Rosa Nasser RHR, Mendonça BB: Effectiveness of treating ovarian hyperstimulation syndrome with cabergoline in two patients with gonadotropin-producing pituitary adenomas. Fertil Steril 2006, 86, 719.e15-18.

[40] Davis A, Goel S, Picolos M, Wang M, Lavis V: Pituitary apoplexy after leuprolide. Pituitary 2006, 9, $263-265$.

\section{Address for correspondence:}

Jowita Halupczok

Department of Endocrinology, Diabetology and Isotope Therapy

Wroclaw Medical University

Pasteura 4

50-367 Wrocław

Poland

E-mail: jowita16@op.pl

Conflict of interest: None declared

Received: 4.04.2014

Revised: 11.06.2014

Accepted: 11.06.2014 